\title{
Wegener's granulomatosis mimicking a parotid abscess
}

\author{
G L Jones, MRCS, A D Lukaris, MRCOPhth*, H V Prabhu, MBBS, DLO ${ }^{\dagger}$, M J K M Brown, FRCS ${ }^{\dagger}$, \\ J BONDESON, MD, PHD
}

\begin{abstract}
We present the case of a previously healthy 59-year-old man who was under treatment for scleritis and episcleritis when he developed a parotid-gland swelling and pus-producing sinus. On surgical exploration, the features were those of a parotid abscess, but the lesion not only failed to heal post-operatively but increased in size very significantly. There was also severe necrotizing keratitis of the eyes. Due to clinical suspicion and a positive antineutrophil cytoplasmic antibodies test, Wegener's granulomatosis was diagnosed and the patient successfully treated with cyclophosphamide and steroids. Previously, a number of cases of Wegener's granulomatosis causing salivary-gland swelling have been reported in the literature; this is the first case in which the disease has masqueraded as a parotid abscess.
\end{abstract}

Key words: Parotid Gland; Wegener's Granulomatosis; Abscess; c-ANCA

\section{Introduction}

Wegener's granulomatosis is a systemic disease that primarily affects the kidneys and respiratory tract. However, being one of the remaining great masqueraders of medicine, it can cause necrotizing inflammation of a number of other sites, frequently involving structures in the head and neck region. Pathologically, it is characterized by a combination of granulomatous vasculitis affecting small to medium-sized blood vessels. Diagnosis may be made from biopsy of the affected organ, but serum analysis for antineutrophil cytoplasmic antibodies (c-ANCA) has considerable sensitivity and specificity to detect the disease. Wegener's granulomatosis is a potentially lethal condition, and early diagnosis and treatment are required to prevent morbidity and mortality.

Wegener's granulomatosis of the head and neck is usually associated with sinus involvement, ulceration of the nasal septum or the external-ear canal, or destruction of the vocal folds. Around 30 cases of salivary-gland enlargement as an initial manifestation of Wegener's granulomatosis have been described, several of them demonstrating the value of c-ANCA to determine the diagnosis. ${ }^{1-11}$ We report here a case with more extensive and rapidly progressive involvement of the parotid than was found in those previously described; the patient also suffered severe autoimmune eye disease, with necrotizing keratitis threatening sight in both eyes.

\section{Case report}

A previously healthy, 59-year-old, nonsmoking, retired male teacher presented to his general practitioner with a painful left eye. He was referred to the ophthalmology department, where episcleritis was diagnosed and treated with prednisolone and ofloxacin eye drops. The treatment was initially successful but the disease became recurrent.

After four months, the patient developed a firm, rightsided preauricular swelling and was referred to the otorhinolaryngology department. Computerized tomography (CT) demonstrated a $3 \mathrm{~cm}$ soft-tissue mass of the superficial right parotid gland. Fine-needle aspiration cytology was inconclusive. Blood investigations, including a full blood count, erythrocyte sedimentation rate (ESR), urea and electrolytes profile, rheumatoid factor, and cANCA and p-ANCA levels, were within normal limits.

Over the next four months, the preauricular swelling developed a sinus discharging saliva and pus. A parotid abscess was suspected and an exploration of the right parotid gland revealed an abscess cavity with the facial nerve involved in its base. The histology agreed with the clinical findings of a chronic parotid abscess; no acid-fast bacilli were isolated.

Post-operatively, an infected, discharging salivary fistula developed which subsequently ulcerated despite vigorous antimicrobial therapy. A biopsy taken from the wound edge showed a haemorrhagic ulcerated surface (Figure 1) with underlying inflammatory infiltration. There was also concentric fibrosis of blood-vessel walls and elastin staining showed vascular damage (see Figure 1 inset). A granulomatous component was seen, with palisaded rows of histiocytes and multinucleate giant cells (Figure 2). The CT of the right parotid was repeated and showed nodes up to $1 \mathrm{~cm}$ in diameter in the right cervical chain and an enlarged left submandibular gland, but no focal collection. Over the next three months, daily dressings of the wound were performed but with little or no evidence of healing.

The parotid ulceration grew, and there was a copious discharge of pus and saliva. By this time, there was also a complete facial palsy. The condition of the eyes also

From the Department of Otorhinolaryngology, Royal Gwent Hospital, Newport, the *Department of Ophthalmology, University Hospital of Wales, Cardiff, the 'Department of Otorhinolaryngology, Royal Gwent Hospital, Newport, and the ${ }^{\ddagger}$ Department of Rheumatology, University Hospital of Wales, Cardiff, Wales.

Accepted for publication: 15 April 2005. 


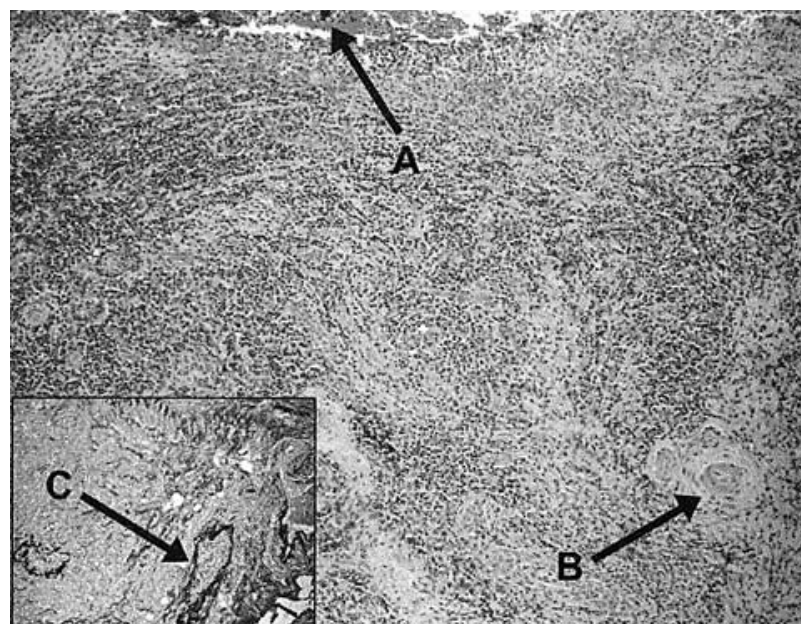

FIG. 1

(A) Ulcerated surface with (B) underlying inflammation and vessels showing concentric perivascular fibrosis (haematoxylin $\&$ eosin; $\times 100)$ and $(C)$ vascular damage (inset: elastin stain).

deteriorated rapidly. The patient developed bilateral mucopurulent discharge resembling a bacterial conjunctivitis. The right eye became the focus of concern when a corneal epithelial defect and conjunctival abscess were noted, most likely secondary to lagophthalmos consequent to the facial-nerve palsy. There was active scleritis and episcleritis, and also rapidly progressive necrotizing keratitis of both eyes, particularly the right one (Figure 2), that threatened the patient's vision. Treatment was commenced with povidone iodine 5 per cent, ofloxacin and penicillin eye drops, but with little or no effect. Vision in the right eye was now blurred to light perception but 6/5-3 vision was retained in the left eye.

Given this sudden and rapid deterioration, a multidisciplinary review was undertaken. Due to the presence of non-neoplastic, necrotizing lesions in two organs, Wegener's granulomatosis was suspected, although the patient lacked all typical symptoms of systemic Wegener's granulomatosis. X-rays of the lungs and sinuses

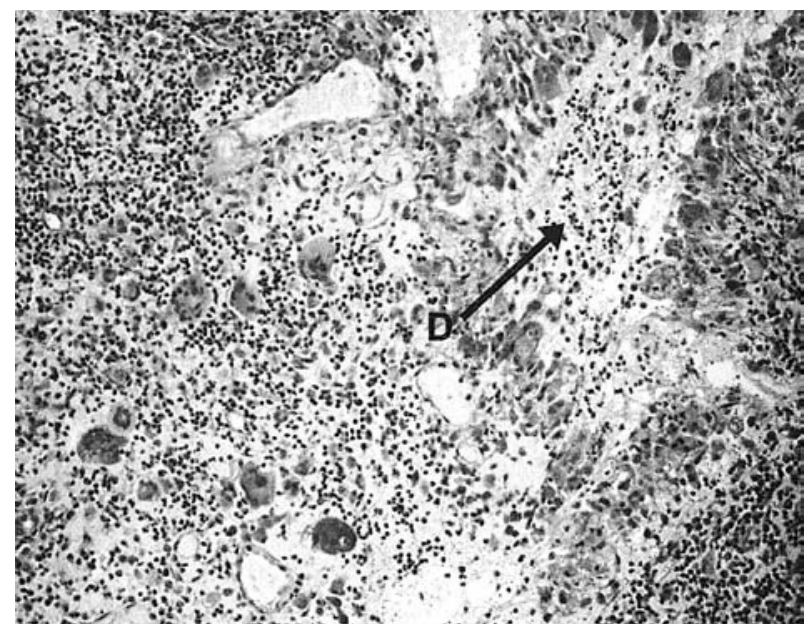

FIG. 2

(D) Palisaded multinucleate histiocytes (periodic acid Schiff; $\times 200$ )

were normal, and the patient had no symptoms indicating compromise of these organs. Renal function was normal, as was urinary sediment; there was no rash or arthritis, nor any vasculitic mononeuritis. The ESR was 55, C-reactive protein 280, and tests for rheumatoid factor and antinuclear antibodies were both negative. Importantly, the c-ANCA was now positive (1/160), and positivity for PR3-ANCA (88.2) confirmed the clinical suspicion of Wegener's granulomatosis.

Treatment commenced immediately with infusions of methylprednisolone $500 \mathrm{mg}$ daily along with six cycles of cyclophosphamide $1-\mathrm{g}$ infusions. The response in the parotid wound was dramatic, as illustrated by Figures $3 \mathrm{a}$ (pre-treatment) and $3 \mathrm{~b}$ (three weeks post-treatment). There was healthy granulation and rapid healing of the parotid ulceration, and this process continued after the patient was placed on oral cyclophosphamide (100 mg daily, reducing to $75 \mathrm{mg}$ daily due to leukopenia) and prednisolone (30 $\mathrm{mg}$ daily, reducing to $5 \mathrm{mg}$ daily), resulting in complete healing of the lesion within four months (Figure 3c). The outcome was equally favourable
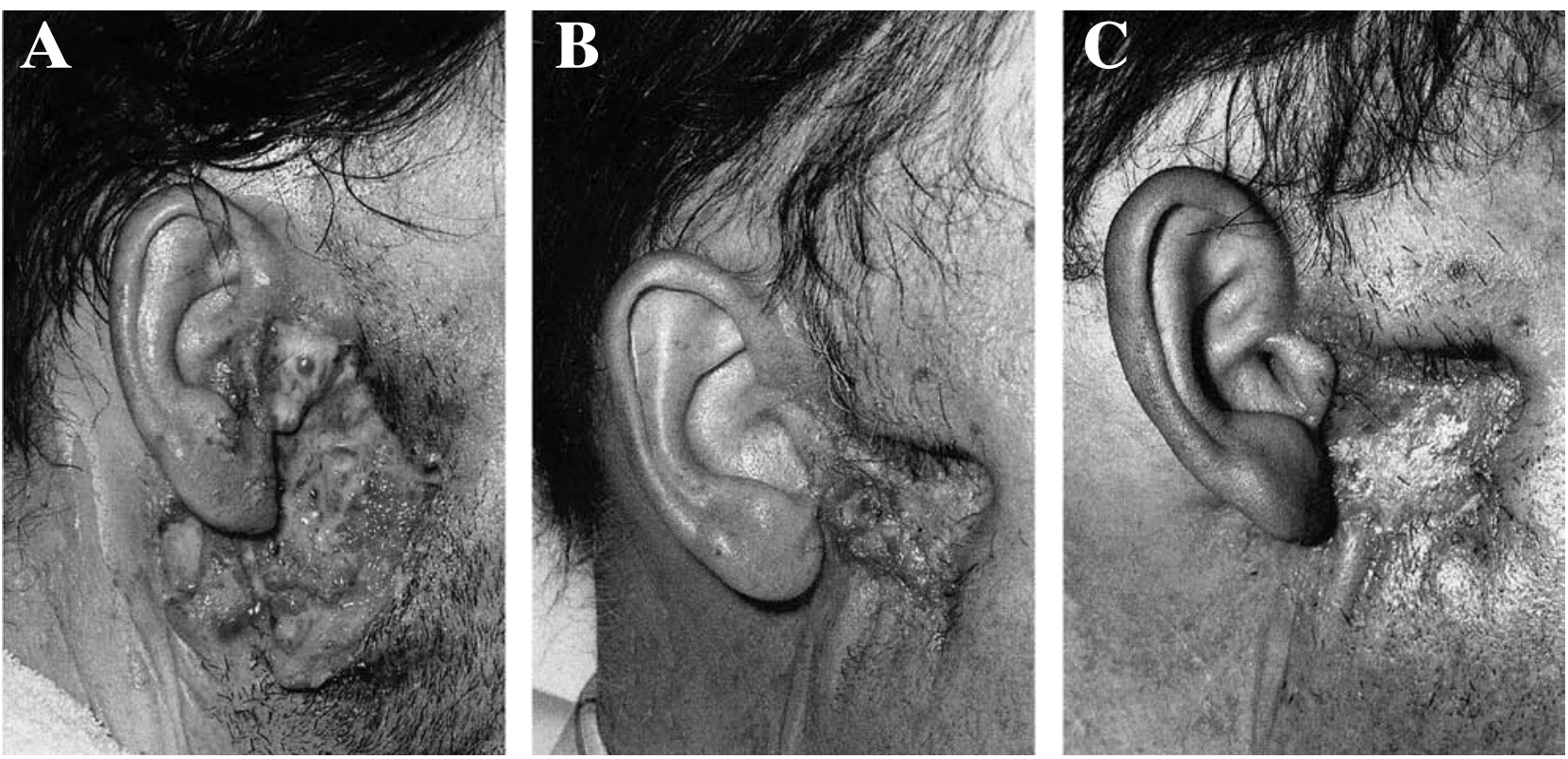

FIG. 3

The patient's parotid lesion, photographed (a) just prior to immunosuppressive treatment, (b) three weeks later, showing healthy granulation, and (c) four months later, showing almost complete healing. 
TABLE I

SUMMARY OF WEGENER'S GRANULOMATOSIS CASES WITH PAROTID INVOLVEMENT

\begin{tabular}{llll}
\hline Reference & Cases $(n)$ & Clinical presentation & Histology \\
\hline Lustmann et al. $(1994)^{3}$ & 1 & Acute transient parotitis & Granulomatous inflammatory process \\
& 1 & Transient parotitis & Not done \\
& 1 & Parotitis & Not done \\
& 1 & Mikulicz disease & Not done \\
& 1 & Parotid nodules & Necrotizing granuloma \\
& 1 & Bilateral parotid masses & Not done \\
& 1 & Pain, parotid mass & Granulomata \\
& 1 & Sjogren's syndrome & Epitheloid cells near necrotic foci \\
& 2 & Parotid enlarged & Acute and chronic inflammation without vasculitis \\
Benson-Mitchell et al. $(1994)^{4}$ & 1 & No information & Giant cells; acute and chronic inflammation \\
Berge et al. $(2000)^{7}$ & 2 & & Poorly formed granulomas; geographic necrosis \\
Saravanappa et al. $(2000)^{8}$ & 1 & Parotid swelling & \\
Bucolo et al. $(2003)^{10}$ & 1 & Painful parotid swelling & Chronic inflammation \\
Bulbol et al. $(2003)^{11}$ & 1 & Painful parotid swelling & Chronic inflammation \\
Liu et al. $(2003)^{12}$ & 1 & Parotid swelling & Cytology suggentive of nonspecific inflammation \\
& & Painful parotid swelling & Necrotizing granulomatous inflammation \\
& & Submandibular swelling & Cytology suggestive of granulomatous process \\
\hline
\end{tabular}

*Lustmann et al reviewed cases of Wegener's granulomatosis in the salivary gland, including 14 cases involving the parotid glands.

with regard to the patient's ocular lesions, although the established corneal melting in the right eye required a corneal transplant. The established facial palsy required further eye surgery and also the fitting of a bandage contact lens. Vision in the left eye was perfect, and a final visual acuity of 6/36 was achieved in the right eye.

Three years after the onset of this gentleman's symptoms, cyclophosphamide was paused for two weeks due to leukopenia; the patient again developed scleritis, which disappeared when cyclophosphamide was reintroduced at a lower dose.

\section{Discussion}

Salivary-gland involvement in Wegener's granulomatosis is an uncommon but well recognized phenomenon. In 1994, Lustmann et al. reviewed 23 cases of Wegener's granulomatosis in salivary glands, ${ }^{3}$ and since then a further nine cases have been reported in the English literature. ${ }^{4-12}$ Of all these cases, 20 involved the parotid gland, and these are summarized in Table I. The other cases involved the submandibular glands only. Characteristically, the salivary gland swelling is initially asymptomatic, although some patients reported pain and ear discomfort. Some cases were unilateral, others bilateral. It is interesting that our patient also had submandibular gland involvement.

Diagnosis is often difficult, since CT and ultrasonography findings are nonspecific. Differential diagnosis includes malignancy, viral or bacterial parotitis, tuberculosis, actinomycosis, lues, sarcoidosis and Sjögren's syndrome. Salivary-gland involvement usually occurs early in the disease course of Wegener's granulomatosis, and although some cases have lung or sinus disease, the majority do not have other obvious signs of systemic disease. The route to diagnosis is twofold: measurement of c-ANCA, and histological findings from salivary-gland biopsies. The problem with the latter route is that not all biopsies demonstrate the cardinal histological features of granulomatosis, necrosis and vasculitis. ${ }^{13,14}$ The highest diagnostic yield is obtained from open-lung biopsies from pulmonary lesions, but in biopsies from the head and neck region only 16 per cent demonstrated all three cardinal features and 23 per cent had two features. Secondly, there can be difficulty distinguishing Wegener's granulomatosis from the histological differentials, which include granulomatous infection, lymphomatoid granulomatosis, idiopathic midline granuloma, allergic angiitis and granulomatosis, sarcoidosis, and foreign body reactions.

Our report is the first documented case of a localized parotid abscess formation caused by Wegener's granulomatosis, probably due to secondary infection of a parotid granuloma. It demonstrates some of the difficulties in making an early diagnosis of this condition. The clinical picture, with a large ulceration in the parotid region, was a most uncommon and misleading one but, fortunately, the presence of autoimmune eye disease raised suspicion of systemic vasculitis. Another difficulty is that c-ANCA can be negative in early disease, as in our case and at least one other involving the parotid, illustrating the value of repeat analysis of this autoantibody when there is clinical suspicion of Wegener's granulomatosis. Neither is our case the only one in which initial biopsies from the affected salivary gland were inconclusive.

The prognosis of the patient with Wegener's granulomatosis of the parotid is highly dependent on an early diagnosis being made of this potentially lethal disease. In the majority of literature cases of Wegener's granulomatosis of the parotid, the prognosis has been favourable, but some have gone on to develop severe renal and central nervous system disease.

- Salivary gland involvement is a rare manifestation of Wegener's granulomatosis

- this case presentation of Wegener's granulomatosis in the parotid gland mimicked an abscess, possibly due to secondary infection of a parotid granuloma

The second abnormal feature in our case was the widespread and serious eye disease, with necrotizing keratitis threatening the patient's vision. This is another uncommon but well recognized manifestation of Wegener's granulomatosis, again often occurring early in the course of the disease. ${ }^{15-17}$ Again, this complication responded well to prednisolone and cyclophosphamide, although the corneal defect required surgical treatment.

This case is thus an example not just of the sometimes misleading presentation of Wegener's granulomatosis, but also of the often favourable response to efficient treatment instituted before irreversible organ damage occurs. 


\section{Acknowledgement}

We would like to thank Dr I Thompson, Consultant Histopathologist (The Royal Gwent Hospital, Newport, Wales) for providing and commenting on the histological slides.

\section{References}

1 Specks U, Colby TV, Olsen KD, Deremee RA. Salivary gland involvement in Wegener's granulomatosis. Arch Otolaryngol 1991;117:218-23

2 Vanhauwaert BG, Roskams TA, Vanneste SB, Knockaert DC. Salivary gland involvement as initial presentation of Wegener's disease. Postgrad Med J 1993;69:643-5

3 Lustmann J, Segal N, Markitziu A. Salivary gland involvement in Wegener's granulomatosis. A case report and review of the literature. Oral Surg Oral Med Oral Pathol 1994;77:254-9

4 Benson-Mitchell R, Tolley N, Croft CB, Roberts D. Wegener's granulomatosis presenting as a unilateral parotid swelling. J Laryngol Otol 1994;108:431-2

5 AhSee KW, McLaren K, Maran AGD. Wegener's granulomatosis presenting as major salivary gland enlargement. J Laryngol Otol 1996;110:691-3

6 Singh S, Kaura D, Kumari S, Gupta D, Dey P, Suri S. Wegener's presenting as submandibular swelling. Sarcoidosis Vasc Diffuse Lung Dis 1997;14:81-2

7 Berge S, Niederhagen B, von Lindern J-J, Appel T, Reich $\mathrm{RH}$. Salivary gland involvement as an initial presentation of Wegener's disease. A case report. Int J Oral Maxillofac Surg 2000;29:450-2

8 Saravanappa N, Bibas A, Singhal A, Davis JP. Unilateral parotid swelling as initial manifestation of Wegener's granulomatosis. J Otolaryngol 2000;29:396-7

9 Crean SJ, Adams R, Bennett J. Sublingual gland involvement in systemic Wegener's granulomatosis: a case report. Int J Oral Maxillofac Surg 2002;31:104-6

10 Bucolo S, Torre V, Montemagno A, Beatrice F. Wegener's granulomatosis presenting with otologic and neurologic symptoms: clinical and pathological correlations. J Oral Pathol Med 2003;32:438-40
11 Bulbol Y, Ozlu T, Oztuna F. Wegener's granulomatosis with parotid gland involvement and pneumothorax. Medical Principles and Practice 2003;12:133-7

12 Liu SY, Vlantis AC, Lee WC. Bilateral parotid and submandibular gland enlargement: rare features of Wegener's granulomatosis. J Laryngol Otol 2003;117:148-50

13 Devaney KO, Travis WD, Hoffman G, Leavitt R, Lebovics $\mathrm{R}$, Fauci AS. Interpretation of head and neck biopsies in Wegener's granulomatosis: a pathologic study of 126 biopsies in 70 patients. Amer J Surg Path 1990;14:555-64

14 Lie JT. Wegener's granulomatosis: histological documentation of common and uncommon manifestations in 216 patients. Vasa 1997;26:261-70

15 Charles SJ, Meyer PAR, Watson PG. Diagnosis and management of systemic Wegeners granulomatosis presenting with anterior ocular inflammation. $\mathrm{Br} \mathrm{J}$ Ophthalmol 1991;75:201-7

16 Messmer EM, Foster CS. Vasculitic ulcerative keratitis. Surv Ophthalmol 199;43:379-96

17 Harper SL, Letko E, Samson CM, Zafirakis P, Sangwan V, Nguyen Q et al. Wegeners granulomatosis: the relationship between ocular and systemic disease. J Rheumatol 2001;28:1025-32

Address for correspondence:

Graeme Lewis Jones,

ENT Registrar,

Singleton Hospital,

Sketty Road,

Swansea, UK.

E-mail: gljones@doctors.org.uk

Mr G L Jones takes responsibility for the integrity of the content of the paper.

Competing interests: None declared 